NOVARTIS

\title{
S1P-S1PR1 activity controls VEGF-A signal- ing during lymphatic vessel development
}

\section{Running title: S1PR1 is required for lymphangio- genesis}

Golding-Ochsenbein AM*, Vidal S, Wilmering Wetter B, Guibourdenche C, Beerli C, Chang L, Leonhard S, Holway N, Seuwen K, Jurisic G*

Novartis Institutes for BioMedical Research, Novartis Pharma AG, Basel, Switzerland

* Corresponding authors 


\section{Abstract}

3 Sphingosine-1-phosphate (S1P), a lipid signaling molecule produced by endothelial cells,

4 is required for development and homeostasis of blood vessels. However, its role during

5 lymphatic vessel development is unclear. We show in murine newborns that pharmaco-

6 logically enhanced S1P signaling increases VEGF-A-dependent LEC proliferation. In con-

7 trast, S1PR1 inhibition, mediated by the antagonist NIBR0213 or LEC-specific genetic

8 deletion of S1pr1, promotes filopodia formation and vessel branching, independent of

9 VEGF-A. To investigate the S1P and VEGF-A signaling crosstalk observed in vivo, we

10 used LECs cultured in vitro. We demonstrate that S1P activates endogenous S1PR1 in

11 a constitutive, autocrine manner. Importantly, S1P-S1PR1 activity was required for

12 VEGF-A-induced LEC proliferation and strongly supported ERK1/2 activation and

13 VEGFR-2 trafficking to the perinuclear area. In conclusion, S1P-S1PR1 signaling pro-

14 motes VEGF-A-dependent LEC proliferation and limits migratory and filopodia-forming

15 responses. Hence, S1P-S1PR1 signaling is required for balanced growth factor-induced

16 lymphangiogenesis and correctly patterned lymphatic vessels during postnatal develop-

17 ment. 


\section{Introduction}

2 Lymphangiogenesis, the formation of new lymphatic vessels from pre-existing ones, is

3 orchestrated by tissue-derived growth factors such as VEGF-C and VEGF-A, and by cell

4 intrinsic mechanisms (Vaahtomeri et al., 2017) that create a balance between proliferating

5 and migratory, filopodia-forming lymphatic endothelial cells (LECs) (James et al., 2013).

6 This balance is key to subsequent vascular patterning events such as branching and

7 hence, influence lymphatic vessel morphology (Gerhardt et al., 2003). Interestingly,

8 VEGF-A is known to enhance proliferation and less so sprouting (Nagy et al., 2002;

9 Wirzenius et al., 2007), whereas VEGF-C induces both sprouting and proliferation.

VEGF-A activates the receptor VEGFR-2, whereas VEGF-C, in its processed form, activates both VEGFR-2 and VEGFR-3. The latter receptor is critically required for lymphangiogenesis. Both receptors belong to the receptor tyrosine kinase family, and ligand activation leads to dimerization and auto-phosphorylation, followed by internalization (reviewed in (Zhang and Simons, 2014)) and fusion with endosomal compartments (Simons et al., 2016; Zhang and Simons, 2014). These events are important for the activation of several downstream signaling pathways including ERK1/2 that is crucial for endothelial cell function (Simons et al., 2016).

Sphingosine-1-phosphate (S1P), a lipid mediator secreted by erythrocytes, platelets and endothelial cells, signals via 5 specific G protein-coupled receptors (S1PR1-5) by inducing their internalization and activating downstream signaling cascades (Mullershausen et al., 2009; Yanagida and Hla, 2017). S1PR1, highly expressed by endothelial cells, is crucial for embryonic blood vessel development and function (Yanagida and Hla, 2017). It 
1 stabilizes endothelial barrier and negatively regulates blood endothelial cell sprouting

2 (Ben Shoham et al., 2012; Bigaud et al., 2016; Gaengel et al., 2012; Jung et al., 2012).

3 However, opposing conclusions of S1P acting either pro- or anti-angiogenic were previ-

4 ously made (Ben Shoham et al., 2012; Caballero et al., 2009; English et al., 2001; English

5 et al., 2000; Gaengel et al., 2012; Licht et al., 2003). For lymphatic vessels, S1P was

6 reported to promote lymphangiogenesis in a matrigel plug assay in adult mice (Yoon et

7 al., 2008). Additionally, sphingosine kinase activity in LECs was shown to be required for

8 generation of S1P and proper lymphatic vessel patterning and arrangement of cell junc-

9 tions in vivo (Pham et al., 2010). However, the mechanism of how S1P affects lymphatic vessel development, in particular its effect on growth factor signaling, and the role of

11 S1PR1 during this process are not known.

12 We show here, for the first time, that S1P-S1PR1 activity influences lymphangiogenesis by promoting VEGF-A-dependent LEC proliferation and by limiting migratory and filopo-

14 dia-forming responses. Furthermore, antagonism of S1PR1 leads to dysfunctional lym15 phatic vessels. Hence, S1P-S1PR1 signaling is required for the correct patterning and 16 maintenance of function of the lymphatic vessel network during postnatal development. 


\section{Methods}

2

3 Endothelial cell culture

$4 \quad$ Primary adult human dermal LECs (PromoCell) or human umbilical vein endothelial cells

5 (HUVECs) (PromoCell) were cultured on plates coated with $50 \mu \mathrm{g} / \mathrm{ml}$ Purecol collagen

6 type I (Advanced Biomartix) and in EGM-MV medium without VEGF-A (PromoCell). For

7 starvation of the cells, EBM-MV (PromoCell) without FCS was used. For washing steps

$8 \mathrm{pH} 7.4$ phosphate-buffered saline (PBS) without $\mathrm{CaCl}_{2}$ and $\mathrm{MgCl}_{2}$ (Gibco) was used. Cells

9 were treated with the dual non ATP-competitive sphingosine kinase inhibitor SKI-II

10 (SIGMA), S1P (Avanti Polar) diluted in 90\% DMSO containing $50 \mathrm{mM} \mathrm{HCl,} \mathrm{NIBR0213}$

11 (Novartis) and recombinant carrier-free human VEGF-A165 (R\&D systems).

Measurements of S1P concentration

14

15

16

17

18

19

20 21 Sph, $0.1 \mathrm{ng} / \mathrm{ml}$ for S1P.

S1P concentrations were measured as previously described (Billich et al., 2013). To reduce unspecific binding, extracts were subjected to acetylation as described in (Berdyshev et al., 2005). Sphingosine derivatives were detected in positive mode; negative ionization was used for S1P. Quantification was performed based on the area ratios of the compound over internal standard in the extracted ion chromatograms. Recovery was $>86 \%$ for all analytes. The limit of quantification, as determined by the lowest calibration sample showing signal-to-noise ratio $>5$ and accuracy $<14 \%$, was $0.5 \mathrm{ng} / \mathrm{ml}$ for 
Electric Cell-substrate Impedance Sensing (ECIS)

2 The lymphatic endothelial cell barrier tightness was assessed using an ECIS $\AA$ ZO instrument with a 16-well array station (Applied Biophysics). 8-well chamber slides (8W10E+, ibidi) were pre-incubated with $50 \mu \mathrm{g} / \mathrm{ml}$ fibronectin (Roche) and then washed with sterile $\mathrm{H}_{2} \mathrm{O}$. Next, 30000 adult human dermal LECs per well were plated in EGM and impedance values were recorded at $4,000 \mathrm{~Hz}$ every 30 seconds. When a monolayer with stable impedance levels of 3000-3500 ohm was formed, treatments with compounds started. To

8 investigate LEC endogenous S1P functions, $10 \mu \mathrm{M}$ SKI-II or DMSO control was spiked into corresponding wells. After $4 \mathrm{~h}$ cells were washed with PBS and starved in EBM containing again $10 \mu \mathrm{M}$ SKI-II for $3 \mathrm{~h}$. Next, cells were treated $\pm 1 \mu \mathrm{M}$ S1P, S1P $+0.3 \mu \mathrm{M}$ NIBR0213 or DMSO control. To rule out NIBR0213 toxic effects, 0.1 or $0.01 \mu \mathrm{M}$ NIBR0213 was spiked into corresponding wells. After 5 h $10 \mu \mathrm{M}$ S1P or DMSO control were spiked into corresponding wells. Graphs were generated using TIBCO Spotfire 6.5.3. Impedance curves were normalized to DMSO control.

Immunofluorescent approaches for in vitro experiments

17 Cells were starved for $4 \mathrm{~h}$ or overnight in medium without FCS and received indicated treatments for indicated times. Then cells were fixed with 4\% PFA for 10 min at room temperature and washed with PBS before blocking for $1 \mathrm{~h}$ in immunomix (PBS containing $5 \%$ donkey serum, $1 \%$ bovine serum albumin (BSA) and $0.1 \%$ Triton-X). The following

21 antibodies were incubated overnight at $4^{\circ} \mathrm{C}$ : rat anti-human CD31 (DAKO, M082329-2), rabbit anti-human S1PR1 (Santa Cruz, sc-25489), goat anti-human VEGFR-2 (R\&D sys- 
1 step in $0.1 \%$ Triton-X PBS, samples were incubated for $1 \mathrm{~h}$ at room temperature with

2 Alexa Fluor 488, 594 or 647 nm-conjugated secondary antibodies (Invitrogen). Samples were imaged with a Zeiss LSM 700 (Zeiss) confocal microscope using the Zen 2011 SP3

4 (black edition) software or for VEGFR-2 trafficking with a Yokogawa CV7000 system us-

5 ing the CellVoyager R1.17.05 software. Yokogawa images were analyzed with ImageJ:

6 The perinuclear area was defined by Hoechst area plus a $1.625 \mu \mathrm{m}$ radius and total cell area was defined based on CD31 plasma membrane staining. Total cell VEGFR-2 was

8 defined as $100 \%$. Values were normalized to DMSO control.

Homogeneous time resolved fluorescence (HTRF)

11 HTRF approaches (Cisbio) were used to determine VEGFR-2 pTyr1175 and ERK 12 pThr202/Tyr204 levels. To measure VEGFR-2 pTyr1175 levels after VEGF-A stimulation with or without S1PR1 pathway modulation, 40000 cells per 96 well were seeded in EGM in the morning. In the evening, cells were washed with PBS and starved overnight in EBM without FCS. Next, cells were stimulated with the indicated treatments for 2 and 5 min and $2 \mathrm{~h}$ before processing according to manufacturer's instructions. For determination of

17 pERK1/2 levels in the presence or absence of SKI-II, 20000 human dermal LECs per 96 well were seeded in EGM. The next day $10 \mu \mathrm{M}$ SKI-II was spiked into corresponding wells and incubated for $4 \mathrm{~h}$ before EGM removal, a PBS wash and the addition of EBM starvation medium without FCS $\pm 10 \mu \mathrm{M}$ SKI-II. Cells were starved for $3 \mathrm{~h}$ before $10 \mathrm{~min}$ stimu-

21 lation with the indicated treatments. Next, cells were processed according to manufac-

22 turer's instructions. Fluorescent signals were measured with TRF Light Unit EnVision 23 (PerkinElmer). EC50 and $E_{\max }$ were calculated with Prism 6 (GraphPad Software). 
2 For the 5-ethynyl-2'-deoxyuridine (EdU) proliferation assay, 5000 LEC per well of a 96

\section{Cell proliferation assay} well plate were seeded in EGM in the morning. In the evening, cells were washed with PBS before an overnight starvation in EBM. The next morning, cells were treated with $100 \mathrm{ng} / \mathrm{ml}$ VEGF-A $\pm 0.3 \mu \mathrm{M}$ NIBR0213 or DMSO control and in the evening $10 \mu \mathrm{M}$ EdU was spiked. $24 \mathrm{~h}$ after the addition of VEGF-A cells were fixed with $4 \%$ PFA before further processing according to the Click-iT EdU Imaging Kit (Invitrogen). Experiments were performed with eight replicates per group. Per well nine non-overlapping images were acquired and analyzed with the Operetta High Content Screening System microscope (PerkinElmer) and Harmony 4.1 software.

\section{Mice}

For in vivo experiments with pharmacological approaches C57BL/6 females were purchased from Charles River Laboratories (France) or came from Novartis Pharma AG Basel internal breeding facilities. For lymphatic vessel-specific depletion of S1PR1, the wellestablished mouse lines Prox-1creERT2 mice (Bazigou et al., 2011) and S1PR1 floxed mice (Allende et al., 2003) were crossed and bred in the animal breeding facility of Novartis Pharma AG, Basel. For pharmacological approaches C57BL/6 pups received i.p. 4 or $20 \mathrm{mg} / \mathrm{kg} /$ day Compound 31 (Cmpd 31) (Novartis) diluted in PEG200/5\% glucose (70/30\%) or PBS/PEG200/5\% glucose $(50 \% / 35 \% / 15 \%)$ for co-treatments, 3 or 6 mg/kg/day VEGF-A neutralizing antibody 4G3 (Novartis) diluted in PBS/PEG200/5\% glucose (50\%/35\%/15\%), 1, 5 or $10 \mathrm{mg} / \mathrm{kg} /$ day NIBR0213 (Novartis) diluted in PEG200/5\% glucose $(70 / 30 \%)$ or PBS/PEG200/5\% glucose (50\%/35\%/15\%) for co-treatments with 
1 4G3 (6 mg/kg/day) from postnatal day (P) 0.5 to P3.5 and were sacrificed at P4.5. To

2 induce recombination events, homozygous S1pr1 flx and Prox-1-CreERT2+ (S1PR1-

$3 \mathrm{i} \triangle \mathrm{LEC}$ ) or Prox-1-CreERT2- (control) littermates received intragastric injections of $50 \mu \mathrm{g}$

4 tamoxifen (Sigma), dissolved in $\mathrm{EtOH}$ and sunflower seed oil (Sigma) from P1 to P4.

5 Tissues were harvested at P7 to allow enough time for recombination to occur.

6

\section{Animal study approval}

All in vivo experiments were approved by the Cantonal Veterinary Office Basel-Stadt (license number BS-2586).

\section{Whole-mount staining and morphometric analyses}

Abdominal skin or diaphragms were harvested and skin samples were incubated for $1 \mathrm{~h}$ in $20 \mathrm{mM}$ EDTA at $37^{\circ} \mathrm{C}$ to facilitate the removal of the epidermis. Then tissues were fixed for $2 \mathrm{~h}$ in freshly prepared $4 \%$ PFA at $4^{\circ} \mathrm{C}$. Next, samples were washed in PBS and blocked in immunomix (described above) for $1 \mathrm{~h}$ at RT before overnight primary antibody incubation at RT. The following primary antibodies were used: rat-anti mouse S1PR1 (R\&D Systems, MAB7089), hamster anti-mouse CD31 (Millipore, MAB1398Z), goat antimouse Prox-1 (R\&D Systems, AF2727), rabbit anti-mouse LYVE1 (AngioBio, 11-034), goat anti-mouse LYVE1 (R\&D Systems, AF2125), rabbit anti-mouse KI-67 (Neomarkers, RM-9106-S1), goat anti-mouse VEGFR-2 (R\&D Systems, AF644). The next day, whole mounts were washed and incubated for 2 hours with AlexaFluor 488, 594 or 647-conjugated secondary antibodies (all from Invitrogen) at RT. Whole-mount z-stacks were acquired on a Zeiss LSM 700 confocal microscope with the Zen 2011 SP3 (black edition) 
1 software. Images were processed with Zen software. For lateral diaphragm segments

2 images were processed with a personalized Fiji macro (Schindelin et al., 2012) (Rueden et al., 2017). The macro automated the following tasks: the maximum intensity projection

4 of each image stack was calculated and then each projection was stitched together using

5 Fiji's Grid/Collection stitching plugin (Preibisch et al., 2009). The stitched image was then

6 thresholded using Fiji's default (IsoData) algorithm (Ridler and Calvard, 1978) and auto-

7 matic threshold value, colored green and then saved. The macro is freely available at

8 https://github.com/Novartis/ExactAddressTBC. Lateral diaphragm segments were ana-

9 lyzed as described previously (Ochsenbein et al., 2016). Other images were analyzed by hand with ImageJ.

To assess growth factor levels in postnatal diaphragms, tissues were collected and mRNA levels were assessed by qPCR. To do so, two diaphragms of P2.5 C57Bl6 pups were collected in RNA later (Qiagen) before they were processed in RLT buffer containing $1 \%$ mercaptoethanol (Sigma) with the help of metal beads (MP Biomedicals) and the FastPrep-24 tissue ruptor (MP Biomedicals). Next, RNA was extracted according to the RNeasy Micro Kit (Qiagen). The RNA was transcribed to cDNA using the High-Capacity cDNA Reverse Transcription Kit (Applied Biosystems). The PCR reactions were per20 formed using a Quant Studio 7 Flex Fast Real-Time PCR System (Applied Biosystems 21 by life technologies) and the following Taqman primers (Thermo Fisher): Actb 22 (Mm02619580_g1), Vegf-A (Mm00437306_m1), Vegf-C (Mm00437310_m1), Vegf-D (Mm01131929_m1). Data is presented by fold change to Actb. 
3 Statistical analyses were performed using Prism 7 (GraphPad Software). Data are shown

4 as mean \pm standard deviation. Normal distribution was tested with Shapiro-Wilk test. Nor-

5 mally distributed data were analyzed using the student's t-test or, for comparisons of

6 means of more groups, one-way ANOVA and Dunnett's test were used. For non-normal

7 distributed data non-parametric tests where used. Statistical significance is indicated by

8 asterisks: ${ }^{*} p<0.05,{ }^{* *} p<0.01,{ }^{* * *} p<0.001,{ }^{* * *} p<0.0001$. Differences were consid-

9 ered statistically significant when $p<0.05$. 


\section{Results}

Increasing tissue S1P levels enhances lymphatic endothelial cell proliferation in vivo in a VEGF-A-dependent manner

To study the role of S1P in early postnatal lymphangiogenesis, we used a previously established murine model where lymphatic vessels on the pleural side of the diaphragmatic muscle are monitored (Ochsenbein et al., 2016). In a first series of experiments we enhanced S1P signaling by treating pups from postnatal day $(P)$ 0.5-3.5 with Cmpd 31, a highly specific S1P lyase inhibitor that increases S1P concentrations in vivo (Weiler et al., 2014). At P4.5, LYVE1+ lymphatic vessels expressed S1PR1, which was also present on some single cells and on CD31+ blood vessels (Fig. 1A). Intraperitoneal injection of 20 $\mathrm{mg} / \mathrm{kg} /$ day of Cmpd 31 significantly increased S1P levels in muscle tissue (Fig. S1A) and, in the diaphragm, lymphatic vessel diameters and lymphatic endothelial cell (LEC) proliferation (assessed as \%KI-67+ cells per PROX-1+ cells) without changing the vessel branching response (Fig 1B-E,G,H). Thus, we conclude that enhanced S1P signaling boosts LEC proliferation during postnatal development.

Next, we asked whether S1P might increase lymphatic vessel diameter and cell proliferation via cross-talk with VEGFR-2, as reported for blood endothelial cells (Gaengel et al., 2012). Indeed, VEGFR-2 is expressed on LYVE-1 positive lymphatic vessels at P2.5 (Fig S1B) and Vegf-a is the most abundantly expressed endothelial growth factor in the postnatal diaphragm at P2.5 (Fig 1F). We therefore treated neonates from P0.5-3.5 with Cmpd 31 (20 mg/kg/day i.p.) with or without $3 \mathrm{mg} / \mathrm{kg} /$ day of $4 \mathrm{G} 3$, a VEGF-A neutralizing antibody (Poor et al., 2014). The analysis of P4.5 LYVE1 immuno-stained diaphragms revealed 
1 that VEGF-A neutralization alone had no detectable effect on lymphatic vessel parame-

2 ters in the present model, as expected from earlier experiments (Zhang et al., 2018).

3 However, VEGF-A neutralization mildly reduced the Cmpd 31-mediated increase in

$4 \quad$ LYVE1 + lymphatic vessel area and entirely abolished both the Cmpd 31-induced enlarge-

5 ment of lymphatic vessel diameter and LEC proliferation (Fig 1B-E,G,H). None of the

6 tested treatments significantly affected vessel branch formation. Cmpd 31 also increased

7 lymphatic vessel area in the abdominal skin with a trend of reduction by co-treatment with

8 4G3 (Fig S1C). Hence, we report that enhanced S1P signaling enlarges lymphatic vessel

9 diameters and boosts LEC proliferation in a VEGF-A-dependent manner in vivo, without

10 affecting vessel branching. pendently of VEGF-A

14 To complement our pharmacological approach of investigating S1P signaling, we used the specific S1PR1 competitive antagonist NIBR0213 (Quancard et al., 2012) during early postnatal lymphangiogenesis. We treated pups from P0.5 to P3.5 i.p. with $10 \mathrm{mg} / \mathrm{kg} / \mathrm{day}$ of NIBR0213 and sacrificed them at P4.5. At this time point, all NIBR0213 -treated pups had developed chylus ascites, indicative of malfunctioning lymphatic vessels (Fig 2A).

19 Interestingly, analyses of diaphragm whole mounts showed that inhibition of S1PR1 in vivo only mildly reduced LEC proliferation and had no effect on LYVE1-positive lymphatic

21 vessel area, the total lymphatic vessel network expansion or the calculated lymphatic 22 vessel diameter (Fig 2B, Fig S2A). However, NIBR0213 led to a strong, dose-dependent 
1 increase in branch formation (Fig 2B, Fig S2B) and enhanced filopodia formation (Fig 2B,

2 Fig S2C), suggesting that S1PR1 activity inhibits LEC migratory, filopodia-forming re-

3 sponses in vivo. Neutralization of VEGF-A did not alter NIBR0213-mediated enhanced

4 branch formation (Fig S2D, E).

5 To demonstrate that the effects of S1PR1 antagonism are indeed intrinsic to LECs we used an inducible genetic loss-of-function approach to delete S1pr1 specifically in this

7 cell type. Homozygous S1pr1 flx and Prox-1-CreERT2+ or Prox-1-CreERT2- littermates were treated intragastrically from P1-4 with $50 \mu \mathrm{g} /$ day tamoxifen and diaphragms were

9 harvested at P7. Analyses of diaphragm whole mounts revealed that, compared to homozygous S1pr1 flx and Prox-1-CreERT2-control pups, S1PR1 was strongly decreased on LYVE1+ lymphatic vessels in homozygous S1pr1 flx and Prox-1-CreERT2+ (S1PR1i $\triangle$ LEC) littermates, while the S1PR1 expression on blood vessels remained intact (Fig S2F). Importantly, the lymphatic endothelial cell-specific S1PR1 loss-of-function recapitulated the phenotypes caused by NIBR0213 treatment. Lymphatic vessel branching and filopodia formation were strongly increased whereas no changes in lymphatic vessel diameter and LEC proliferation were found (Fig 2C, D, Fig S2G). Deficiency of LEC S1PR1 led to a decreased lymphatic network expansion, suggesting that lymphatic vessel growth was inefficient (Fig 2C). Superficial lymphatic vasculature in the dermis likewise showed increased branch formation, implying a systemic and not an organ-specific effect (Fig 2E). Thus, LEC-specific S1PR1 signaling strongly inhibits lymphatic endothelial cell filopodia

21 formation and vessel branching and is required for a balanced expansion and proper 22 function of the lymphatic vasculature during postnatal development. 
$1 \quad$ Lymphatic endothelial cells in vitro exhibit autocrine and constitutive S1P-S1PR1 signal-

2 ing.

3 To study the molecular mechanism by which S1P regulates lymphatic vessel develop-

4 ment, we characterized S1P production and signaling in primary human dermal lymphatic

5 endothelial cells in vitro. Liquid chromatography-mass spectrometry analyses of LEC-

6 conditioned medium showed that LECs secrete high amounts of S1P (Fig 3A). Treatment

7 with SKI-II, an inhibitor of sphingosine kinases $1+2$ that phosphorylate sphingosine to

8 S1P, completely abolished S1P production. Of note, we showed that already plain, un-

9 conditioned commercial endothelial growth medium (PromoCell EGM-MV) contains ca. 6

$10 \mathrm{nM}$ of S1P (Fig 3A) and conclude that active concentration of S1P is constantly present

11 in regular LEC in vitro culture.

12 Next, we assessed whether S1P can act on LECs directly in an autocrine manner by

13 measuring endothelial barrier function, a process known to be modulated by S1P (Xiong

14 and Hla, 2014), using electric cell-substrate impedance sensing (ECIS) with high temporal

15 resolution. Inhibition of autocrine S1P production by SKI-II treatment led to the destabili-

16 zation of the LEC barrier and this effect could be overcome by the addition of exogenous

17 S1P (Fig 3B). We then used the competitive and selective antagonist NIBR0213 and showed that S1P-induced barrier stabilization was fully reversed, demonstrating the involvement of S1PR1. NIBR0213 is not toxic for LECs as excess S1P could compete for 20 S1PR1 binding and overcome NIBR0213-induced barrier opening (Fig S3). Thus, LECs 21 produce S1P that acts via S1PR1 in an autocrine and constitutive signaling loop that 
1 manifests in LEC barrier function regulation. Furthermore, LECs expressed S1PR1 pro-

2 tein that was internalized upon stimulation with a saturating concentration of $1 \mu \mathrm{M}$ exog-

3 enous S1P (Fig 3C).

5 The mitogenic effect of VEGF-A on lymphatic endothelial cells in vitro depends on S1PS1PR1 signaling

In order to gain a better mechanistic understanding of the interaction between VEGF-A

8 and S1P signaling resulting in the enlarged lymphatic vessel diameters and LEC prolifer-

9 ation in vivo, we studied VEGF-A-mediated proliferation of human dermal LECs in vitro in

10 the presence or absence of S1PR1 signaling. VEGF-A at $100 \mathrm{ng} / \mathrm{ml}$ significantly in-

11 creased cell proliferation as determined by EdU incorporation, and strikingly, this effect

12 was completely inhibited by NIBR0213 (Fig 4A). Thus, S1PR1 signaling is required for

13 VEGF-A-mediated LEC proliferation in vitro.

Autocrine S1P-S1PR1 signaling controls VEGF-A-induced ERK1/2 activation.

16

We went on to study growth factor-induced ERK1/2 activation in vitro, a highly relevant signaling node for mitogenesis in general (Simons et al., 2016) and for VEGF-A downstream activity in particular (Wang et al., 2010). Using a high throughput homogeneous time resolved fluorescence (HTRF) approach, we determined the phospho-ERK1/2 (pERK1/2) response after 10 minutes of titrated VEGF-A with and without NIBR0213 (Fig 4B). Indeed, NIBR0213 inhibited pERK1/2 activation mediated by VEGF-A and caused a 
1 significant increase in $\mathrm{EC}_{50}$ of VEGF-A and strongly decreased the Emax of VEGF-A

2 down to $50 \%$ (Table 1). Thus, we conclude that VEGF-A-induced ERK1/2 activation is

3 strongly dependent on S1PR1 signaling. Furthermore, at 10ng/ml VEGF-A, a sub-satu-

4 rating concentration, the addition of exogenous S1P significantly increased growth factor-

5 induced ERK1/2 activation (Fig S4A). Hence, S1P-S1PR1 signaling enhances and

6 S1PR1 antagonism limits VEGF-A-mediated ERK1/2 activation in LECs in vitro.

7 A positive signaling cross-talk between S1PR1 and VEGFR-2 in LECs was unexpected as earlier work in HUVECs suggested a decrease of VEGF-A-induced pERK1/2 levels

9 (Gaengel et al., 2012). We therefore carried out experiments with human umbilical vein endothelial cells (HUVECs) and obtained results similar to the ones reported here for LECs. Addition of exogenous S1P $1 \mathrm{~h}$ prior to or simultaneously with VEGF-A increased pERK1/2 levels and NIBR0213 significantly reduced the signal (Fig S4B).

To further confirm that growth factor-induced ERK1/2 activation depends on autocrine production of S1P we assessed the $\mathrm{pERK} 1 / 2$ response after VEGF-A treatments in the presence of the sphingosine kinase inhibitor SKI-II (Fig 4C). With SKI-II, VEGF-A was not able to induce ERK1/2 activation significantly compared to control. Co-stimulation with exogenous S1P overcame the SKI-II block for VEGF-A and significantly increased pERK1/2 levels compared to VEGF-A alone (Fig 4C). This increase was inhibited by S1PR1 antagonism.

Taken together, these results show that VEGF-A-induced ERK1/2 activation strongly de-

21 pends on autocrine S1P-S1PR1 signaling. 
1 VEGF-A-induced VEGFR-2 trafficking to the perinuclear area depends on S1P-S1PR1

2 activity

3 To investigate how S1P-S1PR1 affects VEGF-A-mediated signaling events, we studied

$4 \quad$ VEGFR-2 phosphorylation and intracellular trafficking following VEGF-A treatment with

5 or without pharmacological S1P pathway modulation. These events, together with recep-

6 tor internalization, were described to be critical for growth factor action (Simons et al.,

7 2016; Wang et al., 2010). Similarly, S1PR1 receptor internalization, trafficking to peri-

8 nuclear area and persistent signaling from intracellular compartments, were demon-

9 strated to be relevant for endothelial barrier regulation (Mullershausen et al., 2009). As shown in Fig. 5A, VEGF-A significantly increased VEGFR-2 Tyr1175 phosphorylation that was unaffected by the S1PR1 antagonist NIBR0213 at all tested time points and VEGF-

12 A concentrations. This indicates that S1P-S1PR1 is not involved in the initial steps of

13 VEGFR-2 signaling, i.e. receptor dimer formation and autophosphorylation. We next as-

14 sessed whether NIBR0213 co-treatment affected VEGFR-2 co-localization with Rab5, an early endosome marker. The co-localization was similar with NIBR0213 co-treatment compared to VEGF-A alone at all tested time points (Fig S5). However, further onward

17 VEGFR-2 trafficking to the perinuclear region was significantly reduced by NIBR0213 cotreatment compared to VEGF-A alone after 10, 20 and 30 min of treatment (Fig 5B, C).

19 These data show that S1P-S1PR1 signaling is not required for VEGFR-2 trafficking to the 


\section{Discussion}

2 Studies have demonstrated that S1P-S1PR1 signaling is required for development and

3 homeostasis of blood vessels (Ben Shoham et al., 2012; Gaengel et al., 2012; Jung et

4 al., 2012). In addition, a signaling cross talk between VEGFR-2 and S1PR1 was reported

5 in blood endothelial cells (Gaengel et al., 2012). However, in the past different groups

6 have drawn differing conclusions on whether S1P has a pro- or anti-angiogenic role (Ben

7 Shoham et al., 2012; Caballero et al., 2009; English et al., 2001; English et al., 2000;

8 Gaengel et al., 2012; Licht et al., 2003). For lymphatic vessels, effect of S1P on patterning

9 was described (Pham et al., 2010), and a matrigel implant in vivo model suggested an overall pro-lymphangiogenic effect (Yoon et al., 2008). While it appeared likely that S1PR1 represents the receptor transducing S1P effects in LECs similar to BECs, there was no clear demonstration of this from earlier studies.

This study shows, for the first time, that S1P-S1PR1 activity affects lymphangiogenesis by promoting VEGF-A-dependent LEC proliferation and by limiting LEC migratory and filopodia-forming responses in vivo. Filopodia formation and proliferation are hallmarks of endothelial tip and stalk cells, respectively. These cell phenotypes are key players in vascular patterning, and thus we suggest that the vascular network morphology is critically dependent on tightly controlled S1P-S1PR1 signaling.

19 While it was previously shown that S1PR1 signaling limits migratory, filopodia-forming responses during blood vessel development (Ben Shoham et al., 2012; Gaengel et al.,

21 2012; Jung et al., 2012), a striking synergistic action of S1P with VEGF-A in stimulating

22 cell proliferation, resulting in lymphatic vessel enlargement in vivo, was not yet shown 
1 and is surprising. As earlier studies demonstrated that VEGF-C/VEGFR-3 signaling is

2 required for lymphangiogenesis during the first days after birth (Karpanen et al., 2006;

3 Ochsenbein et al., 2016), we conclude that elevated S1P amplifies VEGF-A signaling in

4 addition to ongoing VEGF-C growth factor signals. Therefore, under physiological condi-

5 tions, VEGF-C signaling does not saturate LEC proliferation, which can be further en-

6 hanced by combined action of VEGF-A and S1P. In support of this, ectopic expression of

7 VEGF-A was shown to increase proliferation rather than migration and filopodia-formation

8 of LECs (Nagy et al., 2002; Wirzenius et al., 2007). Likewise, neutralization of VEGF-A

9 alone in our developing diaphragm model, in the absence of enhanced S1P signaling, had no effect on assessed morphological parameters. This finding is in line with previ-

11 ously published results (Ochsenbein et al., 2016; Zhang et al., 2018), where genetic or 12 antibody-mediated loss of VEGFR-2 signaling had no impact on postnatal lymphangio13 genesis.

14 Inhibition of S1PR1 signaling with the specific antagonist NIBR0213 led to chylus ascites formation and increased filopodia formation as well as branch formation in vivo, demonstrating dysfunctional and malformed lymphatic vessels. A similar phenotype was obtained in animals where S1PR1 was specifically deleted in LECs, emphasizing the important LEC-intrinsic signaling role of S1P/S1PR1. Of note, our data are well in line with a previous report showing increased filopodia formation in mice with lymphatic endothelial cell-specific depletion of sphingosine kinase, which is needed for the generation of S1P (Pham et al., 2010). Furthermore, as increased filopodia-formation is also seen in S1PR1-deficient blood vessels, our data establish a pan-endothelial mechanism. 
1 For lymphatics we postulate that S1PR1-mediated inhibition of sprouting acts on alter-

2 native growth factor-driven lymphangiogenic processes, independent of VEGF-A. Earlier

3 work showed that VEGF-C is a crucial driver of postnatal lymphangiogenesis (Zarkada et

4 al., 2015; Zhang et al., 2018) and it will be interesting to investigate how S1P and VEGF-

$5 \quad \mathrm{C}$ signaling converge in vivo in the future.

In order to gain better mechanistic insight into how S1P-S1PR1 affects VEGF-A-mediated

7 lymphangiogenic processes we observed in vivo, we characterized S1P-S1PR1 signaling

in LECs in vitro. We confirmed that S1P-S1PR1 signals in a constitutive and autocrine

9 fashion, as expected from earlier genetic studies showing that LECs generate S1P in a sphingosine kinase 1/2 -dependent manner in mice (Pham et al., 2010). Surprisingly, our in vitro data clearly showed that VEGF-A can only signal and exert its mitogenic function when autocrine, constitutive S1P-S1PR1 signaling is active in LECs. The VEGF-A-mediated proliferative response of LECs was completely inhibited following blockade of S1PR1. In line with this finding, antagonizing S1PR1 also strongly inhibited VEGF-A stimulated ERK1/2 activity. Interestingly, 50\% of VEGF-A-induced ERK1/2 activity seems to be S1PR1-independent. However, the proliferation data demonstrate that S1PR1-independent ERK1/2 activation is not sufficient to translate into a functional output. with S1P co-treatment (Gaengel et al., 2012). In our experience, HUVECs responded in

20 a similar way to LECs by increasing the pERK1/2 levels. Taken together, we propose that

21 S1P-S1PR1 signaling is a constitutive, LEC-intrinsic mechanism enhancing VEGF-A sig-

22 naling and proliferative function. In line with this conclusion, sphingosine kinases were 
1 shown to be important for VEGF-A-mediated ERK1/2 activation in retinal blood endothe-

2 lial cells (Maines et al., 2006) and in a bladder tumor cell line (Shu et al., 2002).

3 Upon VEGF-A stimulation, VEGFR-2 is internalized and traffics through different intracel-

4 lular compartments, such as Rab5+ endosomes (Simons et al., 2016). These steps are

5 important for VEGF-A-mediated downstream signaling and aberrant trafficking of VEGFR-2 was shown to decrease ERK1/2 activation (Lanahan et al., 2010). As deficient S1PR1 signaling decreased VEGFR-2 trafficking to the perinuclear region, we speculate that S1PR1-mediated VEGFR-2 trafficking is needed for efficient ERK1/2 activation. Fur-

9 thermore, as S1PR1 antagonism does not impact VEGFR-2 trafficking to Rab5+ early endosomal compartments, we conclude that the crosstalk between VEGFR-2 and S1PR1 signaling happens between early endosomes and compartments in the perinuclear region. Interestingly, others have shown that S1PR1 and VEGFR-2 can form signaling complexes (Bergelin et al., 2010), and both VEGFR-2 and S1PR1 have been shown to traffic through the same (Rab5+) endosomal compartments (Kofler et al., 2018; MartinezMorales et al., 2018) relevant for ERK1/2 signaling (Kofler et al., 2018). Furthermore, sphingosine kinases play an important role in endocytotic membrane trafficking in general (Lima et al., 2017; Shen et al., 2014).

Interestingly, similar hyperproliferative lymphatic vessel phenotypes as the ones induced by enhanced S1P signaling in our study were described in mice with deficient negative 20 regulators of ERK1/2 signaling, namely SPRED1 and RASA1, as well as for gain-of-func21 tion of RAF1, a positive regulator of ERK1/2 (Deng et al., 2013; Lapinski et al., 2012; 22 Taniguchi et al., 2007). Further, lymphatic-specific deletion of the transcription factors 
1 activated ERK1/2 that resulted in lymphatic vessel enlargement without changes in

2 branching (Fatima et al., 2016). Considering these findings together with our finding that

3 S1P-S1PR1 controls VEGF-A-induced ERK1/2 activation, we suggest that S1P acts as

4 an enhancer of the Ras-Raf-MEK-ERK1/2 signaling cascade upon VEGF-A stimulation

5 in vivo, and that negative modulation of S1P-S1PR1 signaling may be beneficial in treat-

$6 \quad$ ing certain genetic diseases linked to lymphatic hyperproliferation.

7 In summary, we show for the first time that autocrine S1P-S1PR1 influences lymphangi-

8 ogenesis by promoting VEGF-A-dependent LEC proliferation and by limiting migratory

9 and filopodia-forming responses. By doing so, S1P-S1PR1 signaling may favor genera-

10 tion of proliferative stalk cells over filopodia-forming tip cells. In line with this notion,

11 S1PR1 negative blood endothelial cells preferably acquire a tip cell position in the growing

12 retinal vasculature (Gaengel et al., 2012). The novel mechanistic insight into the modula-

13 tion of VEGF-A-mediated lymphangiogenesis provided here could be relevant for the un-

14 derstanding of diseases characterized by prominent hyperplastic lymphatic vessels and 15 loss of lymphatic function such as RASopathies. 


\section{Author contributions}

2 A.M.G.O., G.J. and K.S. designed experiments and A.M.G.O. wrote the paper with sup-

3 port of G.J. and K.S.; G.J. and K.S. supervised the study; A.M.G.O., B.W., S.V. and C.B.

4 performed experiments and A.M.G.O. analyzed data; C.G., L.C., S.L. supported perfor-

5 mance of experiments and experimental design; N.H. wrote the personalized Fiji macro

6 and helped with picture processing. 


\section{Acknowledgements}

2 The authors thank Samuel Barbieri, Dominic Trojer, Isabelle Claerr and the Novartis lab

3 animal services for their support with experimental procedures, Dr. Andreas Billich, Dr.

4 Frederic Bassilana, Dr. Marc Bigaud, Dr. Laure Bouchez and Prof. Michael Detmar for

5 scientific input, Dr. Anne Granger for critical reading of the manuscript and the Novartis

6 postdoc program and Dr. Tewis Bouwmeester for their guidance. 
bioRxiv preprint doi: https://doi.org/10.1101/845396; this version posted November 17, 2019. The copyright holder for this preprint (which was not certified by peer review) is the author/funder, who has granted bioRxiv a license to display the preprint in perpetuity. It is made available under aCC-BY-NC-ND 4.0 International license.

\section{Funding}

2 This work was supported by the Novartis Institutes for Biomedical Research. 
Allende, M. L., Yamashita, T. and Proia, R. L. (2003). G-protein-coupled receptor S1P1 acts within endothelial cells to regulate vascular maturation. Blood 102, 3665-3667.

Bazigou, E., Lyons, O. T., Smith, A., Venn, G. E., Cope, C., Brown, N. A. and Makinen, T. (2011). Genes regulating lymphangiogenesis control venous valve formation and maintenance in mice. J Clin Invest 121, 2984-2992.

Ben Shoham, A., Malkinson, G., Krief, S., Shwartz, Y., Ely, Y., Ferrara, N., Yaniv, K. and Zelzer, E. (2012). S1P1 inhibits sprouting angiogenesis during vascular development. Development 139, 3859-3869.

Berdyshev, E. V., Gorshkova, I. A., Garcia, J. G., Natarajan, V. and Hubbard, W. C. (2005). Quantitative analysis of sphingoid base-1-phosphates as bisacetylated derivatives by liquid chromatography-tandem mass spectrometry. Anal Biochem 339, 129-136.

Bergelin, N., Lof, C., Balthasar, S., Kalhori, V. and Tornquist, K. (2010). S1P1 and VEGFR2 form a signaling complex with extracellularly regulated kinase $1 / 2$ and protein kinase C-alpha regulating ML-1 thyroid carcinoma cell migration. Endocrinology 151, 29943005.

Bigaud, M., Dincer, Z., Bollbuck, B., Dawson, J., Beckmann, N., Beerli, C., Fishli-Cavelti, G., Nahler, M., Angst, D., Janser, P., et al. (2016). Pathophysiological Consequences of a Break in S1P1-Dependent Homeostasis of Vascular Permeability Revealed by S1P1 Competitive Antagonism. PLoS One 11, e0168252.

Billich, A., Baumruker, T., Beerli, C., Bigaud, M., Bruns, C., Calzascia, T., Isken, A., Kinzel, B., Loetscher, E., Metzler, B., et al. (2013). Partial deficiency of sphingosine-1phosphate lyase confers protection in experimental autoimmune encephalomyelitis. PLoS One 8, e59630.

Caballero, S., Swaney, J., Moreno, K., Afzal, A., Kielczewski, J., Stoller, G., Cavalli, A., Garland, W., Hansen, G., Sabbadini, R., et al. (2009). Anti-sphingosine-1-phosphate monoclonal antibodies inhibit angiogenesis and sub-retinal fibrosis in a murine model of laser-induced choroidal neovascularization. Exp Eye Res 88, 367-377.

Deng, Y., Atri, D., Eichmann, A. and Simons, M. (2013). Endothelial ERK signaling controls lymphatic fate specification. J Clin Invest 123, 1202-1215.

English, D., Garcia, J. G. and Brindley, D. N. (2001). Platelet-released phospholipids link haemostasis and angiogenesis. Cardiovasc Res 49, 588-599.

English, D., Welch, Z., Kovala, A. T., Harvey, K., Volpert, O. V., Brindley, D. N. and Garcia, J. G. (2000). Sphingosine 1-phosphate released from platelets during clotting accounts for the potent endothelial cell chemotactic activity of blood serum and provides a novel link between hemostasis and angiogenesis. FASEB J 14, 2255-2265.

Fatima, A., Wang, Y., Uchida, Y., Norden, P., Liu, T., Culver, A., Dietz, W. H., Culver, F., Millay, M., Mukouyama, Y. S., et al. (2016). Foxc1 and Foxc2 deletion causes abnormal lymphangiogenesis and correlates with ERK hyperactivation. J Clin Invest 126, 2437-2451.

Gaengel, K., Niaudet, C., Hagikura, K., Lavina, B., Muhl, L., Hofmann, J. J., Ebarasi, L., Nystrom, S., Rymo, S., Chen, L. L., et al. (2012). The sphingosine-1-phosphate receptor S1PR1 restricts sprouting angiogenesis by regulating the interplay between VEcadherin and VEGFR2. Dev Cell 23, 587-599.

Gerhardt, H., Golding, M., Fruttiger, M., Ruhrberg, C., Lundkvist, A., Abramsson, A., Jeltsch, M., Mitchell, C., Alitalo, K., Shima, D., et al. (2003). VEGF guides angiogenic sprouting utilizing endothelial tip cell filopodia. J Cell Biol 161, 1163-1177. 
James, J. M., Nalbandian, A. and Mukouyama, Y. S. (2013). TGFbeta signaling is required for sprouting lymphangiogenesis during lymphatic network development in the skin. Development 140, 3903-3914.

Jung, B., Obinata, H., Galvani, S., Mendelson, K., Ding, B. S., Skoura, A., Kinzel, B., Brinkmann, V., Rafii, S., Evans, T., et al. (2012). Flow-regulated endothelial S1P receptor-1 signaling sustains vascular development. Dev Cell 23, 600-610.

Karpanen, T., Wirzenius, M., Makinen, T., Veikkola, T., Haisma, H. J., Achen, M. G., Stacker, S. A., Pytowski, B., Yla-Herttuala, S. and Alitalo, K. (2006). Lymphangiogenic growth factor responsiveness is modulated by postnatal lymphatic vessel maturation. Am J Pathol 169, 708-718.

Kofler, N., Corti, F., Rivera-Molina, F., Deng, Y., Toomre, D. and Simons, M. (2018). The Rab-effector protein RABEP2 regulates endosomal trafficking to mediate vascular endothelial growth factor receptor-2 (VEGFR2)-dependent signaling. Journal of Biological Chemistry 293, 4805-4817.

Lanahan, A. A., Hermans, K., Claes, F., Kerley-Hamilton, J. S., Zhuang, Z. W., Giordano, F. J., Carmeliet, P. and Simons, M. (2010). VEGF receptor 2 endocytic trafficking regulates arterial morphogenesis. Dev Cell 18, 713-724.

Lapinski, P. E., Kwon, S., Lubeck, B. A., Wilkinson, J. E., Srinivasan, R. S., SevickMuraca, E. and King, P. D. (2012). RASA1 maintains the lymphatic vasculature in a quiescent functional state in mice. $J$ Clin Invest 122, 733-747.

Licht, T., Tsirulnikov, L., Reuveni, H., Yarnitzky, T. and Ben-Sasson, S. A. (2003). Induction of pro-angiogenic signaling by a synthetic peptide derived from the second intracellular loop of S1P3 (EDG3). Blood 102, 2099-2107.

Lima, S., Milstien, S. and Spiegel, S. (2017). Sphingosine and Sphingosine Kinase 1 Involvement in Endocytic Membrane Trafficking. J Biol Chem 292, 3074-3088.

Maines, L. W., French, K. J., Wolpert, E. B., Antonetti, D. A. and Smith, C. D. (2006). Pharmacologic manipulation of sphingosine kinase in retinal endothelial cells: implications for angiogenic ocular diseases. Invest Ophthalmol Vis Sci 47, 5022-5031.

Martinez-Morales, J. C., Romero-Avila, M. T., Reyes-Cruz, G. and Garcia-Sainz, J. A. (2018). S1P1 receptor phosphorylation, internalization, and interaction with Rab proteins: effects of sphingosine 1-phosphate, FTY720-P, phorbol esters, and paroxetine. Biosci Rep 38.

Mullershausen, F., Zecri, F., Cetin, C., Billich, A., Guerini, D. and Seuwen, K. (2009). Persistent signaling induced by FTY720-phosphate is mediated by internalized S1P1 receptors. Nat Chem Biol 5, 428-434.

Nagy, J. A., Vasile, E., Feng, D., Sundberg, C., Brown, L. F., Detmar, M. J., Lawitts, J. A., Benjamin, L., Tan, X., Manseau, E. J., et al. (2002). Vascular permeability factor/vascular endothelial growth factor induces lymphangiogenesis as well as angiogenesis. J Exp Med 196, 1497-1506.

Ochsenbein, A. M., Karaman, S., Proulx, S. T., Goldmann, R., Chittazhathu, J., Dasargyri, A., Chong, C., Leroux, J. C., Stanley, E. R. and Detmar, M. (2016). Regulation of lymphangiogenesis in the diaphragm by macrophages and VEGFR-3 signaling. Angiogenesis 19, 513-524.

Pham, T. H., Baluk, P., Xu, Y., Grigorova, I., Bankovich, A. J., Pappu, R., Coughlin, S. R., McDonald, D. M., Schwab, S. R. and Cyster, J. G. (2010). Lymphatic endothelial cell sphingosine kinase activity is required for lymphocyte egress and lymphatic patterning. $J$ Exp Med 207, 17-27.

Poor, S. H., Qiu, Y., Fassbender, E. S., Shen, S., Woolfenden, A., Delpero, A., Kim, Y., Buchanan, N., Gebuhr, T. C., Hanks, S. M., et al. (2014). Reliability of the mouse model of choroidal neovascularization induced by laser photocoagulation. Invest Ophthalmol Vis Sci 55, 6525-6534. 
Preibisch, S., Saalfeld, S. and Tomancak, P. (2009). Globally optimal stitching of tiled 3D microscopic image acquisitions. Bioinformatics 25, 1463-1465.

Quancard, J., Bollbuck, B., Janser, P., Angst, D., Berst, F., Buehlmayer, P., Streiff, M., Beerli, C., Brinkmann, V., Guerini, D., et al. (2012). A potent and selective S1P(1) antagonist with efficacy in experimental autoimmune encephalomyelitis. Chem Biol 19, 1142-1151.

Ridler, T. W. and Calvard, S. (1978). Picture Thresholding Using an Iterative Selection Method. leee T Syst Man Cyb 8, 630-632.

Rueden, C. T., Schindelin, J., Hiner, M. C., DeZonia, B. E., Walter, A. E., Arena, E. T. and Eliceiri, K. W. (2017). ImageJ2: ImageJ for the next generation of scientific image data. BMC Bioinformatics 18, 529.

Schindelin, J., Arganda-Carreras, l., Frise, E., Kaynig, V., Longair, M., Pietzsch, T., Preibisch, S., Rueden, C., Saalfeld, S., Schmid, B., et al. (2012). Fiji: an open-source platform for biological-image analysis. Nat Methods 9, 676-682.

Shen, H. Y., Giordano, F., Wu, Y. M., Chan, J., Zhu, C., Milosevic, I., Wu, X. D., Yao, K., Chen, B., Baumgart, T., et al. (2014). Coupling between endocytosis and sphingosine kinase 1 recruitment. Nature Cell Biology 16, 652-+.

Shu, X., Wu, W., Mosteller, R. D. and Broek, D. (2002). Sphingosine kinase mediates vascular endothelial growth factor-induced activation of ras and mitogen-activated protein kinases. Mol Cell Biol 22, 7758-7768.

Simons, M., Gordon, E. and Claesson-Welsh, L. (2016). Mechanisms and regulation of endothelial VEGF receptor signalling. Nat Rev Mol Cell Biol 17, 611-625.

Taniguchi, K., Kohno, R., Ayada, T., Kato, R., Ichiyama, K., Morisada, T., Oike, Y., Yonemitsu, Y., Maehara, Y. and Yoshimura, A. (2007). Spreds are essential for embryonic lymphangiogenesis by regulating vascular endothelial growth factor receptor 3 signaling. Mol Cell Biol 27, 4541-4550.

Vaahtomeri, K., Karaman, S., Makinen, T. and Alitalo, K. (2017). Lymphangiogenesis guidance by paracrine and pericellular factors. Genes Dev 31, 1615-1634.

Wang, Y., Nakayama, M., Pitulescu, M. E., Schmidt, T. S., Bochenek, M. L., Sakakibara, A., Adams, S., Davy, A., Deutsch, U., Luthi, U., et al. (2010). Ephrin-B2 controls VEGFinduced angiogenesis and lymphangiogenesis. Nature 465, 483-486.

Weiler, S., Braendlin, N., Beerli, C., Bergsdorf, C., Schubart, A., Srinivas, H., Oberhauser, B. and Billich, A. (2014). Orally active 7-substituted (4-benzylphthalazin-1-yl)-2methylpiperazin-1-yl]nicotinonitriles as active-site inhibitors of sphingosine 1-phosphate lyase for the treatment of multiple sclerosis. J Med Chem 57, 5074-5084.

Wirzenius, M., Tammela, T., Uutela, M., He, Y., Odorisio, T., Zambruno, G., Nagy, J. A., Dvorak, H. F., Yla-Herttuala, S., Shibuya, M., et al. (2007). Distinct vascular endothelial growth factor signals for lymphatic vessel enlargement and sprouting. $J$ Exp Med 204, 1431-1440.

Xiong, Y. and Hla, T. (2014). S1P control of endothelial integrity. Curr Top Microbiol Immunol 378, 85-105.

Yanagida, K. and Hla, T. (2017). Vascular and Immunobiology of the Circulatory Sphingosine 1-Phosphate Gradient. Annu Rev Physiol 79, 67-91.

Yoon, C. M., Hong, B. S., Moon, H. G., Lim, S., Suh, P. G., Kim, Y. K., Chae, C. B. and Gho, Y. S. (2008). Sphingosine-1-phosphate promotes lymphangiogenesis by stimulating S1P1/Gi/PLC/Ca2+ signaling pathways. Blood 112, 1129-1138.

Zarkada, G., Heinolainen, K., Makinen, T., Kubota, Y. and Alitalo, K. (2015). VEGFR3 does not sustain retinal angiogenesis without VEGFR2. P Natl Acad Sci USA 112, 761-766.

Zhang, X. and Simons, M. (2014). Receptor Tyrosine Kinases Endocytosis in Endothelium Biology and Signaling. Arterioscl Throm Vas 34, 1831-1837. 
1 Zhang, Y., Ulvmar, M. H., Stanczuk, L., Martinez-Corral, I., Frye, M., Alitalo, K. and

2

3

Makinen, T. (2018). Heterogeneity in VEGFR3 levels drives lymphatic vessel 

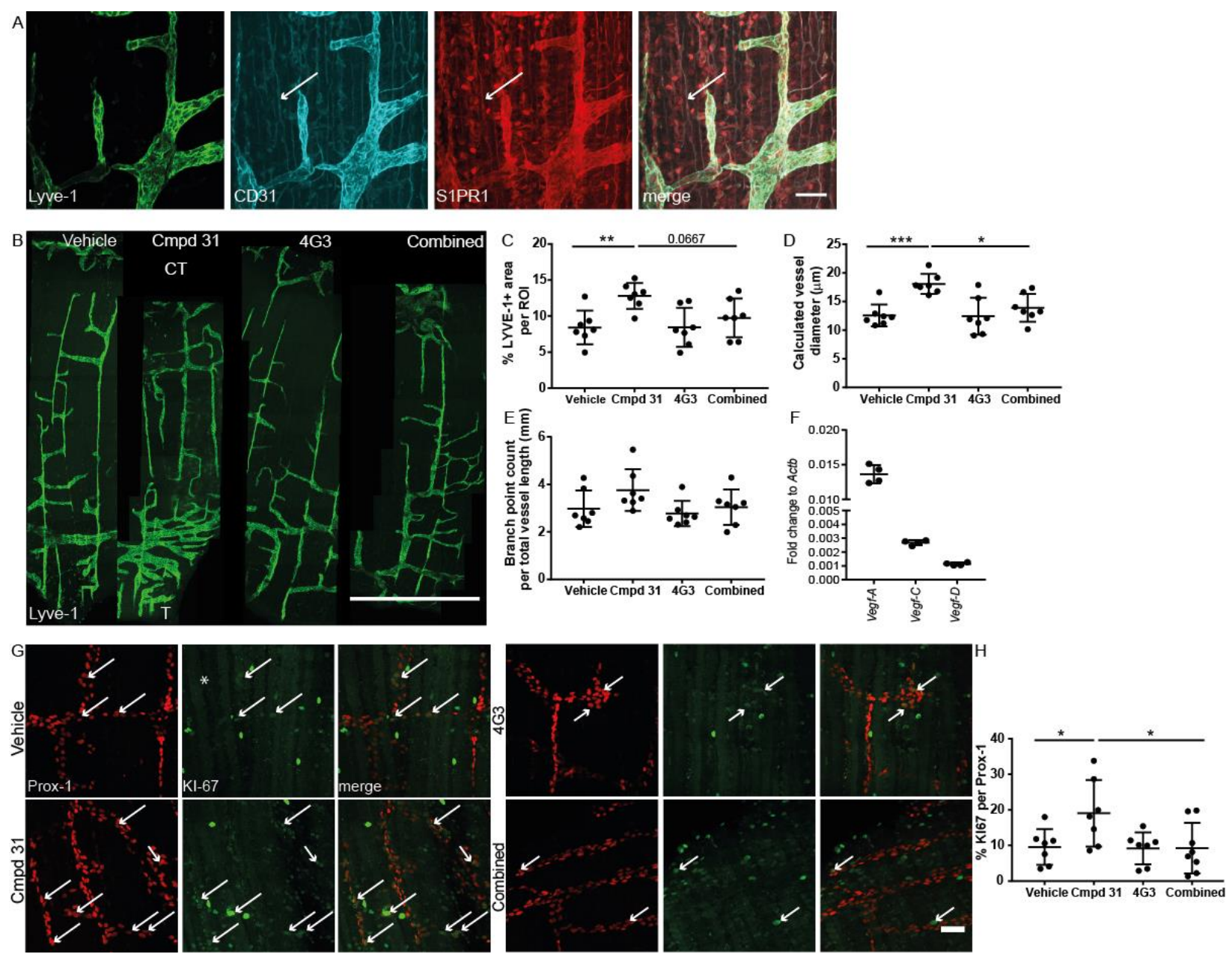

Figure 1: S1P enhances LEC proliferation in a VEGF-A-dependent manner in vivo.

A) Representative confocal images of P4.5 diaphragm whole mounts (pleural side) stained for LYVE1 (green), CD31 (blue) and S1PR1 (red). Arrow highlights an example of CD31+ and S1PR1+ blood vessel. Scale bar $50 \mu \mathrm{m}$. B) Representative stitched confocal images of P4.5 LYVE1-immunostained diaphragm whole mounts (pleural side) of pups exposed to indicated treatments. $\mathrm{T}=$ Thorax wall, CT $=$ Central tendon, scale bar 1 $\mathrm{mm}$. C-E) Quantification of lymphatic vessel parameters of treated pups. F) Real-time qPCR analysis of lymphatic growth factor gene expressions compared to Actb in P2.5 diaphragms. G-H) Representative confocal images and quantifications of diaphragm whole mounts immunostained for Prox-1 (red) and KI-67 (green) after indicated treatments. Arrows: KI-67 + LECs, scale bar $50 \mu \mathrm{m}$. Statistical analyses: Dots represent animal mean, lines represent group means, error bars represent $\pm S D$. Statistical significance was calculated with one-way ANOVA and Dunnett's test. 

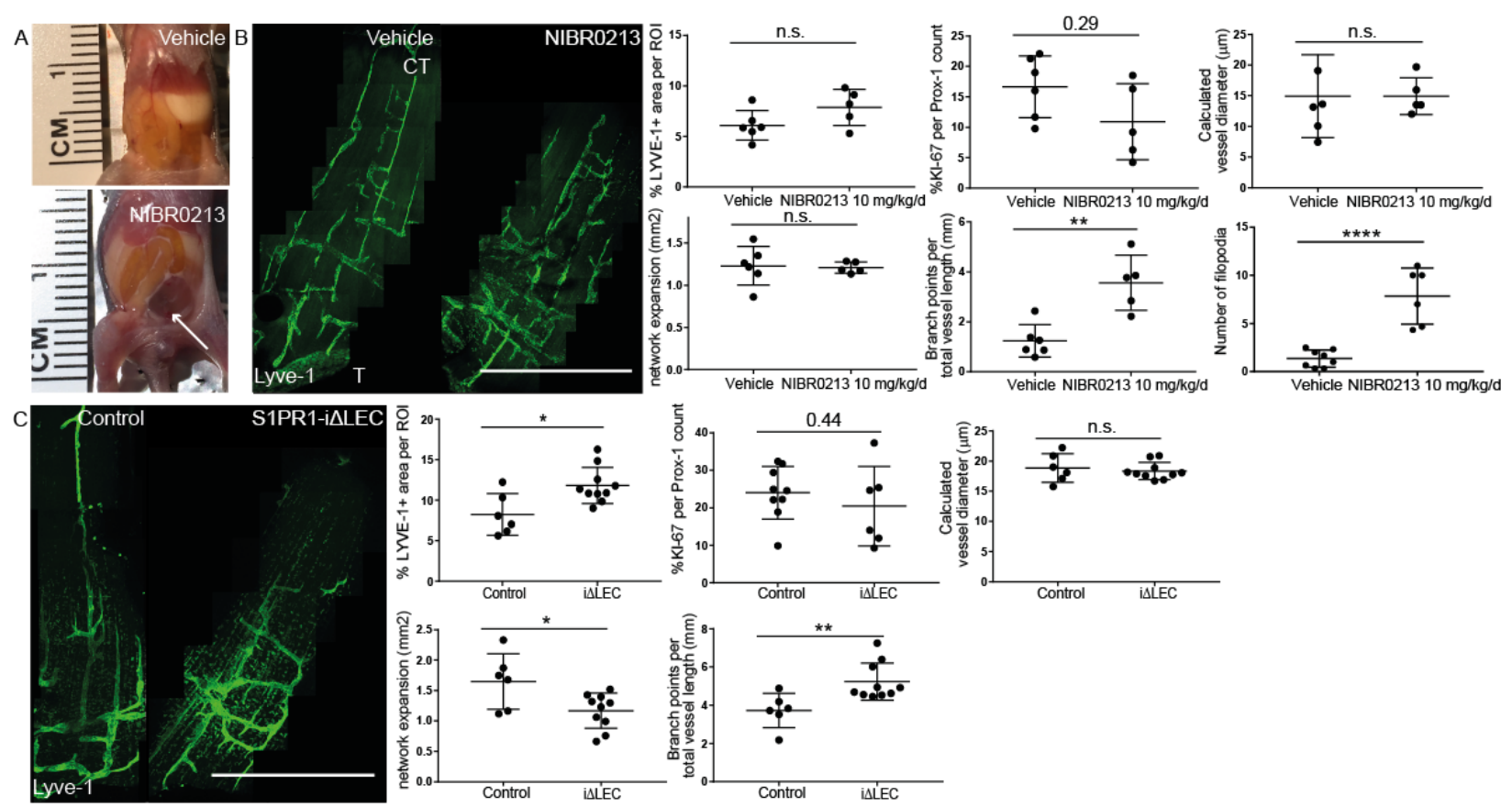

Vehicle NIBR0213 $10 \mathrm{mg} / \mathrm{kg} / \mathrm{d}$
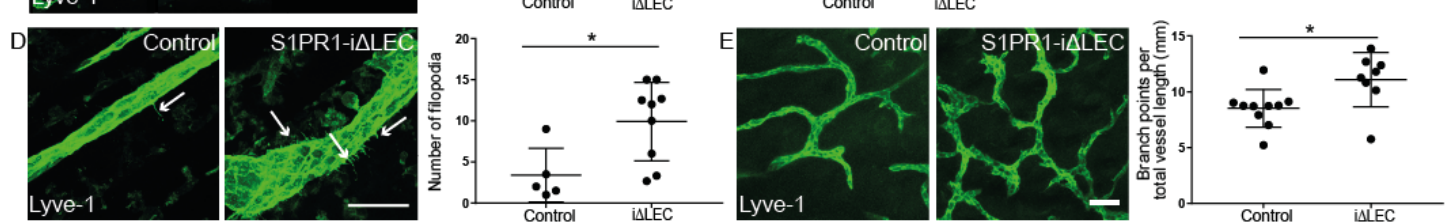

Figure 2: S1PR1 in lymphatic endothelial cells reduces filopodia formation and branching. A) Images of P4.5 vehicle and NIBR0213 treated pups with exposed peritoneum. Arrow indicates chylus ascites. B) Stitched confocal images and quantification of lateral segments of LYVE1 immunostained diaphragm whole mounts of vehicle or 10 $\mathrm{mg} / \mathrm{kg} / \mathrm{day}$ NIBR0213 treated pups. CT= Central tendon, $\mathrm{T}=$ Thorax wall. Scale bar 1 $\mathrm{mm}$. C) Stitched confocal images and quantification of a lateral segment of LYVE1 immunostained diaphragm whole mounts of P7 control or S1PR1-i $\triangle$ LEC pups. Scale bar 1 $\mathrm{mm}$. D) High magnification confocal images and quantification of increased filopodia formation of LYVE1 immunostained diaphragmatic lymphatic vessel in control and S1PR1i $\triangle$ LEC pups. Arrow indicates filopodia, scale bar $50 \mu \mathrm{m}$. E) Confocal images and quantification of LYVE1 immunostained abdominal skin whole mount of P7 control or S1PR1i $\triangle$ LEC pups. Scale bar $50 \mu \mathrm{m}$. Statistical analysis: Dots represent animal mean, lines represent group means, error bars $= \pm S D$. Statistical significance was calculated using an unpaired Student's t-test, or for C Branch points per vessel length a Mann Whitney test. 

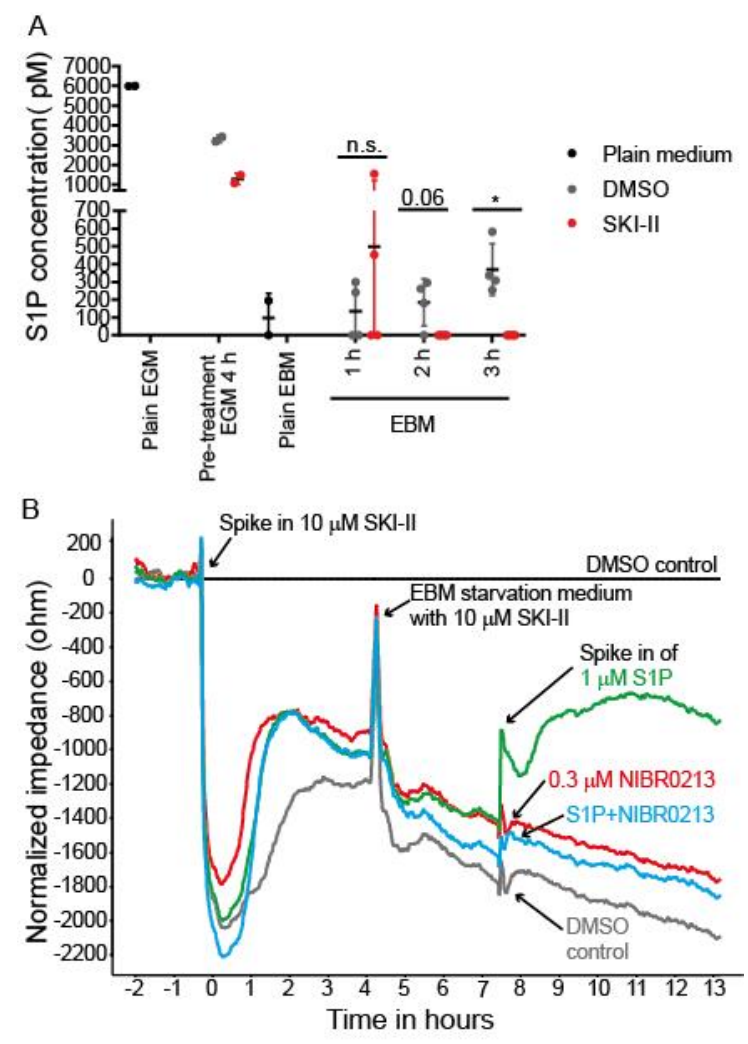

C

CD31

S1PR1

merge
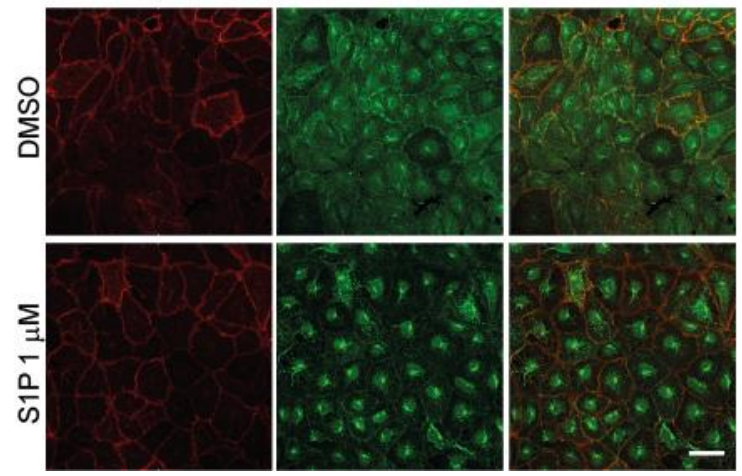

Fig 3: Lymphatic endothelial cells in vitro exhibit autocrine and constitutive S1PS1PR1 signaling. A) S1P concentrations in plain medium (black dots) or in conditioned supernatants of LEC treated \pm SKI-II (grey or red dots). B) Normalized (to DMSO control) electrical impedance of LEC monolayers after indicated treatments. C) CD31 (red) and S1PR1 (green) immunofluorescence of LECs monolayer treated with $1 \mu \mathrm{M} \mathrm{S1P}$ for $1 \mathrm{~h}$. Representative confocal images; Scale bar $=50 \mu \mathrm{m}$. Statistical analyses: Dots represent individual measurements, lines group means, error bars $= \pm S D$. Statistical significance was calculated using an unpaired Student's t-test and Welch's correction for unequal variances. 

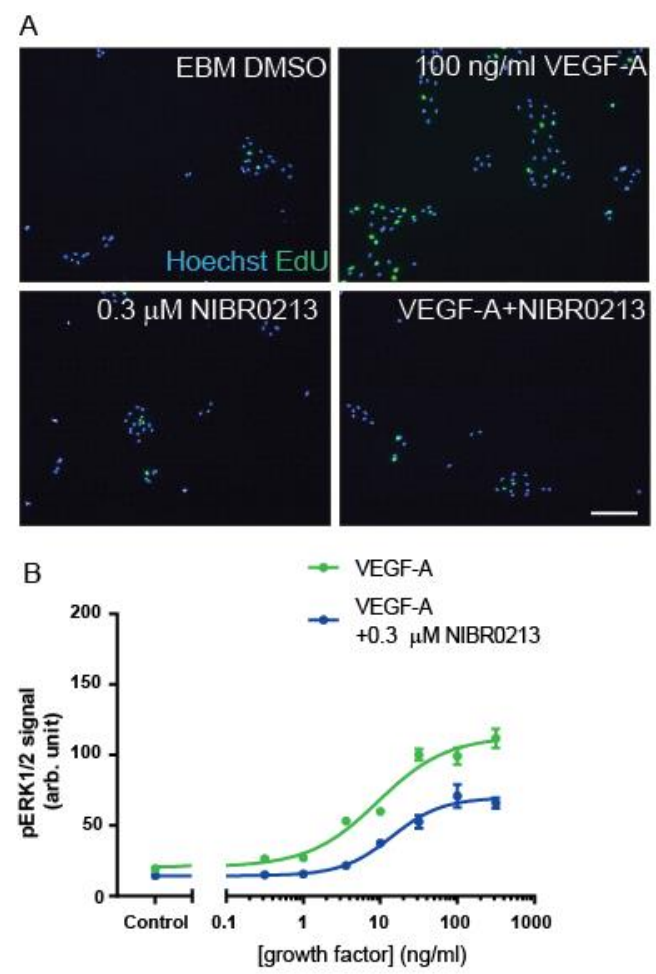
ANOVA and Dunnett's test.
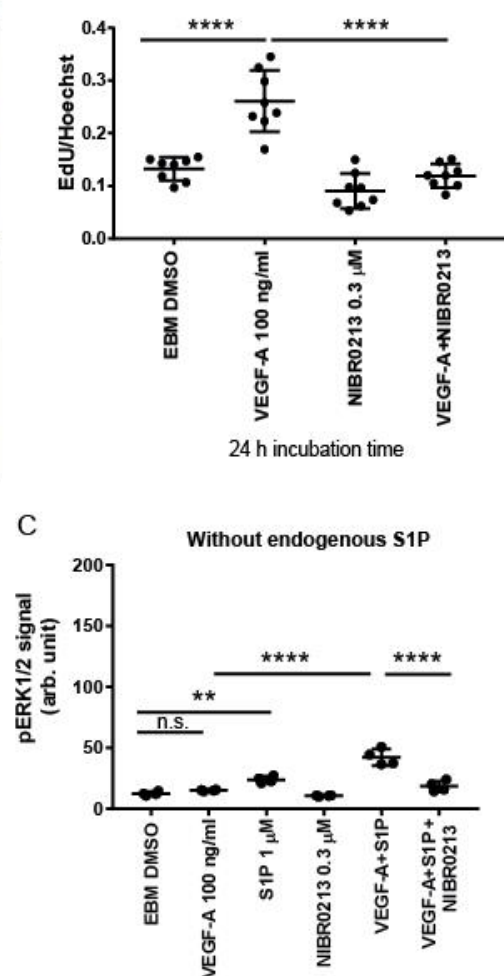

Figure 4: VEGF-A-induced ERK1/2 activation and proliferation depend on autocrine S1P-S1PR1. A) EdU (green) and Hoechst (blue) staining along with quantifications of cultured LEC after $24 \mathrm{~h}$ of indicated treatments. Representative images; Scale bar: 200 $\mu \mathrm{m} . \mathrm{B}, \mathrm{C})$ Quantification of $\mathrm{pERK} 1 / 2$ levels assessed by HTRF in LEC lysates after 10 min of indicated treatments. Statistical analyses: In $A$ and $C$, dots represent individual measurements, lines represent group means. In B, dots represent group means, lines represent fitted curves. Error bars $= \pm S D$. Statistical significance was calculated using one-way 

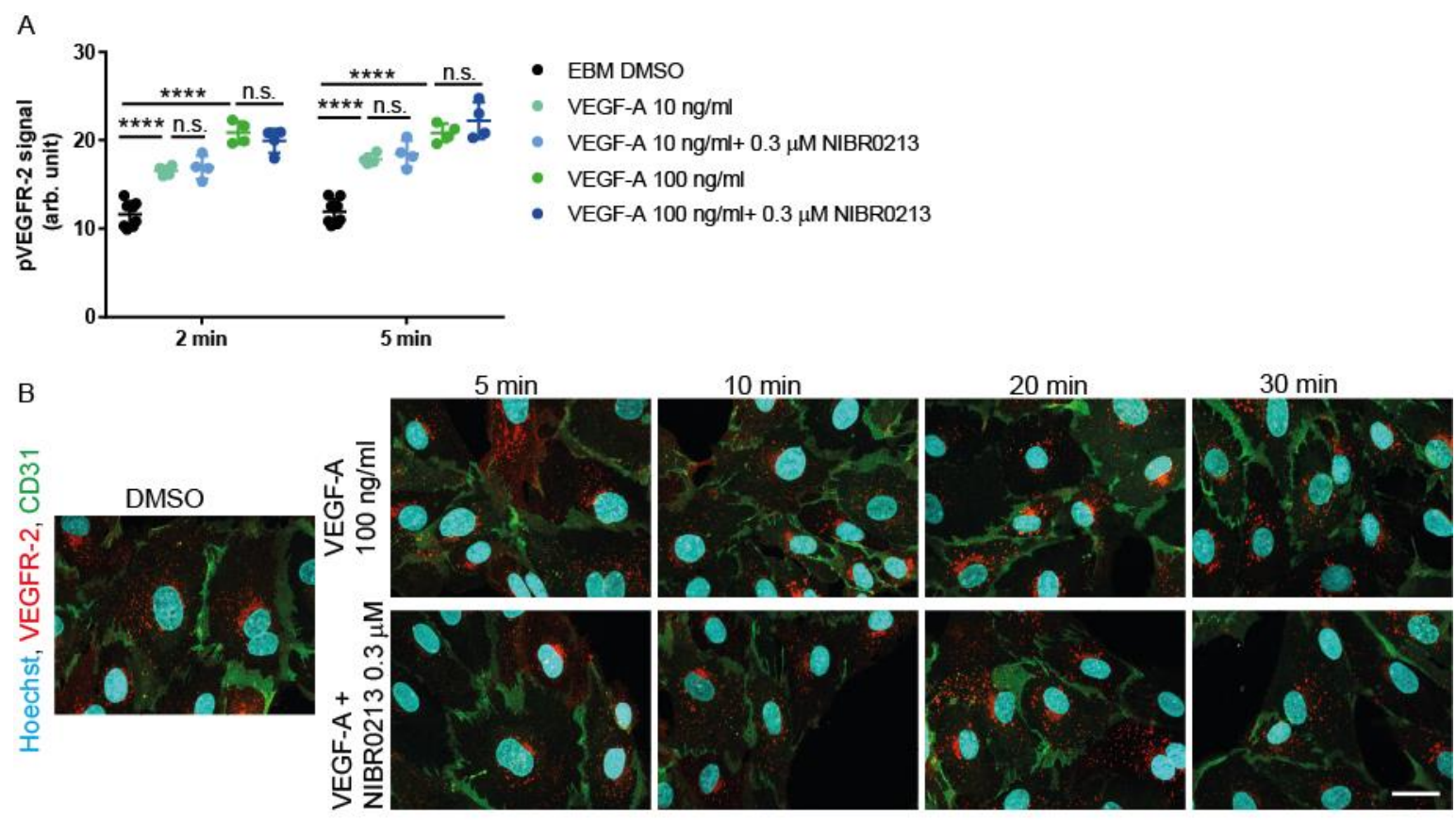

C

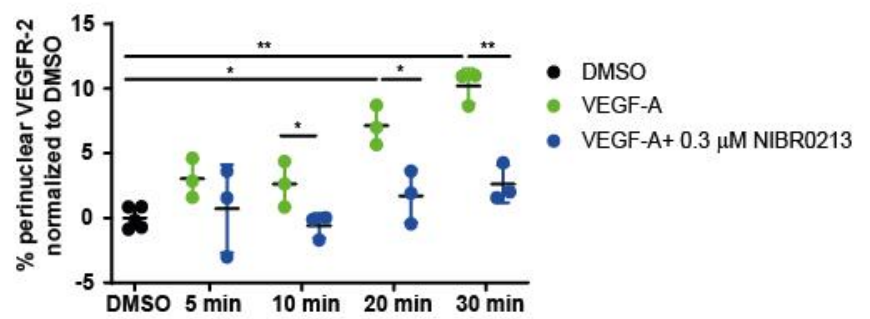

Figure 5: VEGF-A-induced VEGFR-2 translocation to the perinuclear area depends on S1PR1. A) Quantification of pVEGFR-2 levels, assessed by HTRF, in LEC lysates after indicated treatments and incubation times. B,C) Immunofluorescent staining of VEGFR-2 (red), CD31 (green) and Hoechst (blue) and quantification of VEGFR-2 trafficking to the perinuclear area of cultured LEC after indicated treatments and incubation times. Representative confocal images; Scale bars: $50 \mu \mathrm{m}$. Statistical analyses: Dots represent individual measurements, lines group means, error bars $= \pm S D$. Statistical significance was calculated using one-way ANOVA and Dunnett's test. 


\section{Tables}

2 Table 1: Potency and efficacy parameters for VEGF-A induced ERK1/2 activation.

\begin{tabular}{|c|c|c|c|c|}
$\begin{array}{c}\text { Growth factor } \\
\pm 0.3 \mu \mathrm{M} \\
\text { NIBR0213 }\end{array}$ & $\begin{array}{c}\mathrm{EC}_{50}(\mathbf{n g} / \mathbf{m l}) \\
\text { Average } \pm \text { SD, (n) }\end{array}$ & $\begin{array}{c}\text { P value } \\
\text { (one -way ANOVA) }\end{array}$ & $\begin{array}{c}\text { EMax } \\
\text { (\% of VEGF-A) } \\
\text { Average } \pm S D,(n)\end{array}$ & $\begin{array}{c}\text { P value } \\
\text { (one -way ANOVA) }\end{array}$ \\
$\begin{array}{c}\text { VEGF-A } \\
\begin{array}{c}\text { VEGF-A+ } \\
\text { NIBR0213 }\end{array}\end{array}$ & $20.29 \pm 7.61,(3)$ & To VEGF-A: 0.0418 & $55 \pm 11,(5)$ & To VEGF-A: 0.0048
\end{tabular}

3 Representative fitted curves shown in Fig 4B. 